\title{
CENP-B-mediated genome-wide surveillance
}

Eukaryotic genomes have to protect their integrity from the invasion and spread of transposable elements. RNAi-based mechanisms have been shown to fulfill this function in the germline, and new work by Cam and colleagues indicates that centromeric protein CENP-B homologues might have an analogous role in somatic cells, by recruiting chromatin modifiers to repress existing transposable elements and blocking homologous recombination to prevent new mobile-element integration into the host genome.

Working in fission yeast, Cam et al. first showed that two of the three CENP-B homologues, Abp1 and $\mathrm{Cbh} 1$, localize to numerous locations throughout the genome, in particular to the $5^{\prime}$ and $3^{\prime}$ long terminal repeats (LTRs) of Tf 2 retrotransposons, solo LTRs and LTRs associated with ORFs. When Abp1 is genetically removed, expression of Tf2 retrotransposons and LTRassociated genes is elevated. Genetic and biochemical analyses revealed

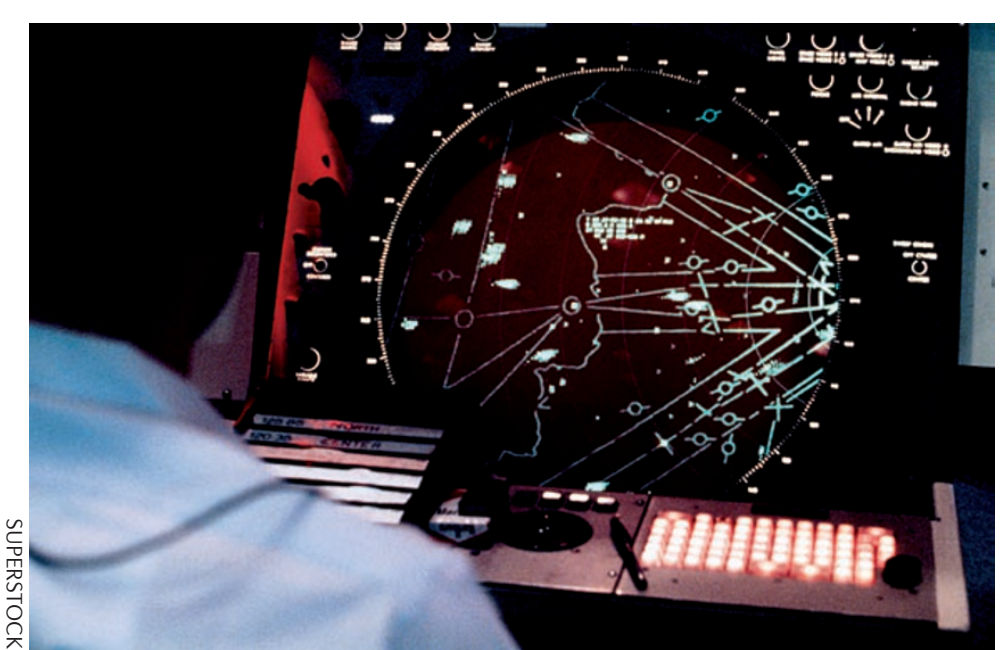

that the silencing function of Abp1 is, in part, mediated by recruiting two histone deacetylases, $\mathrm{Clr} 3$ and Clr6, which are also involved in heterochromatic silencing in different parts of the genome, including centromeres. Moreover, fluorescence in situ hybridization analysis revealed that Tf2 elements dispersed across the genome are organized into three-dimensional clusters within the nucleus. The authors referred to these clusters as Tf bodies, and they are formed less well in CENP-B mutants.

Abp1 and Cbh1 also interact with LTRs of an extinct retrotransposon, Tf1. In its active form, Tf 1 can insert itself into the genome through integrase-mediated transposition or homologous recombination. Cam et al. show that Abp1 and Cbh1 proteins block homologous recombination of an experimentallyintroduced Tf 1 with pre-existing Tf2 retrotransposons in the genome. The suggestion that CENP-Bs prevent retrotransposon integration through homologous recombination was confirmed by sequencing of integration sites.

Consistent with the Tf2 data, transcription and transposition of genome-integrated Tf1 elements are suppressed by the CENP-B homologues; moreover, integration of Tf 1 into a euchromatic gene alters its location within the nucleus, moving it into the Tf bodies.

Altogether, these results show that the CENP-B-based genome surveillance mechanism operates at the level of chromatin-based repression and recombination suppression, which share parallels with heterochromatinmediated silencing. CENP-B homologues are derived from transposases of pogo DNA transposons (found in Drosophila melanogaster), and the authors speculate that during the course of evolution these proteins might have acquired the ability to target retrotransposon LTRs and were subsequently harnessed by the host to control transposable elements and facilitate three-dimensional organization of the genome. Because LTRbound CENP-Bs can repress nearby genes and CENP-Bs might recruit other factors at different genomic locations, the roles of these proteins might also include regulation of other chromatin-related processes such as transcription and DNA replication. Magdalena Skipper

ORIGINAL RESEARCH PAPER Cam, H. P. et al. Host genome surveillance for retrotransposons by transposon-derived proteins. Nature 19 December 2007 (doi:10.1038/nature06499) FURTHER READING Slotkin, R. K. 8 Martienssen, R. Transposable elements and the epigenetic regulation of the genome. Nature Rev. Genet. 8, 272-285 (2007) | Grewal, S. I. \& Jia, S. Heterochromatin revisited. Nature Rev. Genet. 8, 35-46 (2007) 